KEK-TH-554

hep-ph/9801406

January 1998

\title{
Impacts on searching for signatures of new physics from $K^{+} \rightarrow \pi^{+} \nu \bar{\nu}$ decay
}

\author{
Gi-Chol Cho⿴囗十 \\ Theory Group, KEK, Tsukuba, Ibaraki 305, Japan
}

\begin{abstract}
We study impacts on new physics search from the rare decay $K^{+} \rightarrow \pi^{+} \nu \bar{\nu}$. In a certain class of new physics models, the extra contributions to FCNC processes can be parametrized by its ratio to the standard model (SM) contribution with the common CKM factors. The ratio $R_{1}$ has been used in the analysis of $x_{d}$ and $\epsilon_{K}$ parameters. In the above class of models, the $K^{+} \rightarrow \pi^{+} \nu \bar{\nu}$ decay amplitude can be parametrized by the ratio $R_{2}$. Then the experimentally allowed region for new physics contributions can be given in terms of $R_{1}, R_{2}$ and the CP violating phase $\delta$ of the CKM matrix. Constraints on $R_{1}$ and $\cos \delta$ are obtained by taking account of current experimental data and theoretical uncertainties on $B^{0}-\overline{B^{0}}$ and $K^{0}-\overline{K^{0}}$ mixings. We study impacts of future improved measurements by using $\left(R_{1}, R_{2}, \cos \delta\right)$ basis. We discuss contributions in the minimal supersymmetric SM and the two Higgs doublet model as examples.
\end{abstract}

\footnotetext{
${ }^{a}$ Research Fellow of the Japan Society for the Promotion of Science
} 


\section{Introduction}

Processes mediated by flavor changing neutral current (FCNC) have been considered as good probes of physics beyond the standard model (SM). By using the experimentally well measured processes, an existence of new physics may arise as violation of the unitarity of the Cabbibo-Kobayashi-Maskawa (CKM) matrix. Such signatures of new physics will be explored through the determination of the unitarity triangle at B-factories at KEK and SLAC in the near future.

The rare decay $K^{+} \rightarrow \pi^{+} \nu \bar{\nu}$ is one of the most promising processes to extract clean informations about the CKM matrix elements [1]. The decay rate has small theoretical uncertainties because the interactions are dominated by the short-distance physics. The long-distance contributions have been estimated as $10^{-3}$ smaller than the short-distance contributions [2]. The importance of this decay mode on the determination of the unitarity triangle has been discussed in [3, 4]. Furthermore, by carefully examining the consistencies of the CKM matrix elements measured from this decay process and the other processes, we may find a signature of new physics.

Recently, E787 collaboration reported the first observation of an event consistent with this decay process, and obtained $\operatorname{Br}\left(K^{+} \rightarrow \pi^{+} \nu \bar{\nu}\right)=4.2_{-3.5}^{+9.7} \times 10^{-10}[5]$. Although there is still only one candidate event, the report motivates us to examine the implication of the above estimate of the branching fraction and of its improvement in the near future.

In this paper, we study impacts on the search for a new physics signal from the $K^{+} \rightarrow \pi^{+} \nu \bar{\nu}$ decay in a class of new physics models that satisfy the following two conditions: (i) The flavor mixing in the new physics sector is governed by the SM CKM matrix elements, (ii) The main contributions to the FCNC processes are given through loop effects mediated by the third generation particles. The condition (i) means that the effective Lagrangian of the FCNC processes in the new physics sector can be described by the same form with that of the SM besides the estimation of the loop contributions. The condition (ii) implies either the extra contributions from the first two generations do not differ so much, or those are negligible as compared with the contribution from the third generation.

Our assumptions can be valid not only in $K^{+} \rightarrow \pi^{+} \nu \bar{\nu}$ decay but also in other FCNC processes, such as $B^{0}-\overline{B^{0}}$ and $K^{0}-\overline{K^{0}}$ mixings. We will show that new physics contributions to those processes can be parametrized by two quantities, $R_{1}$ for $B^{0}-\overline{B^{0}}, K^{0}-\overline{K^{0}}$ mixings and $R_{2}$ for $K^{+} \rightarrow \pi^{+} \nu \bar{\nu}$ decay. Both quan- 
tities are defined as the ratio of the new physics contribution to that of the SM. Taking account of current experimental data on $x_{d}$ and $\epsilon_{K}$ parameters in $B^{0}-\overline{B^{0}}$ and $K^{0}-\overline{K^{0}}$ mixings, and uncertainties in the hadronic parameters, we show constraints on the new physics contributions in terms of $R_{1}$ and $\cos \delta$, where $\delta$ is the $\mathrm{CP}$ violating phase of the CKM matrix in the standard parametrization [6]. We also find constraints on $R_{1}, R_{2}$ and $\cos \delta$ by assuming the future improvement of the $\operatorname{Br}\left(K^{+} \rightarrow \pi^{+} \nu \bar{\nu}\right)$ measurements. As examples of new physics models which naturally satisfy the conditions (i) and (ii), we examine the consequences of the minimal supersymmetric standard model (MSSM) [7] and the two Higgs doublet model (THDM) 8].

\section{New physics contributions to the FCNC pro- cesses in the $B$ and $K$ meson systems}

The effective Lagrangian for the $K^{+} \rightarrow \pi^{+} \nu \bar{\nu}$ process in the SM is given by [9]:

$$
\mathcal{L}_{\text {eff }}^{K^{+}}=\frac{G_{F}}{\sqrt{2}} \frac{2 \alpha\left(m_{Z}\right)}{\pi} \frac{1}{\sin ^{2} \theta_{W}} \bar{\nu}_{\ell} \gamma^{\mu} P_{L} \nu_{\ell} \bar{s} \gamma_{\mu} P_{L} d \sum_{i=2,3} V_{i 2}^{*} V_{i 1} \eta_{i} D_{W}(i),
$$

where $i$ and $\ell$ are the generation indices for the up-type quarks and leptons, respectively. The CKM matrix element is given by $V_{i j}$ and the projection operator $P_{L}$ is defined as $P_{L} \equiv\left(1-\gamma_{5}\right) / 2$. The QCD correction factor and the loop function are denoted by $\eta_{i}$ and $D_{W}(i)$, respectively. The top quark loop function is given as [9]:

$$
D_{W}(3)=\frac{x_{t}}{8}\left\{\frac{x_{t}+2}{x_{t}-1}+\frac{3 x_{t}-6}{\left(x_{t}-1\right)^{2}} \ln x_{t}\right\}
$$

where $x_{t}=m_{t}^{2} / m_{W}^{2}$. The corresponding QCD correction factor has been estimated as $\eta_{3}=0.985$ for $170 \mathrm{GeV} \leq m_{t} \leq 190 \mathrm{GeV}$ 10. The charm quark loop function with the QCD correction is numerically given as $\eta_{2} D_{W}(2)=\lambda^{4} \times(0.40 \pm 0.06)$ [] where $\lambda \equiv\left|V_{12}\right|$. The error is due to uncertainties in the charm quark mass and higher order QCD corrections. Then, summing up the three generations of neutrino, the branching ratio is expressed as [11]

$$
\begin{aligned}
\operatorname{Br}\left(K^{+} \rightarrow \pi^{+} \nu \bar{\nu}\right)= & 3 \times \frac{G_{F}^{2}}{192 \pi^{3}}\left(\frac{\alpha\left(m_{Z}\right)}{2 \pi \sin ^{2} \theta_{W}}\right)^{2}\left|f_{+}^{K^{+} \pi^{+}}(0)\right|^{2} I\left(m_{K^{+}}, m_{\pi^{+}}\right) \tau_{K^{+}} \\
& \times\left|V_{32}^{*} V_{31} \eta_{3} D_{W}(3)+V_{22}^{*} V_{21} \eta_{2} D_{W}(2)\right|^{2} \\
= & 1.57 \times 10^{-4}\left|V_{32}^{*} V_{31} \eta_{3} D_{W}(3)+V_{22}^{*} V_{21} \eta_{2} D_{W}(2)\right|^{2}
\end{aligned}
$$


where $\tau_{K^{+}}$denotes the lifetime of the $K^{+}$meson. The function $I\left(m_{K^{+}}, m_{\pi^{+}}\right)$gives the phase space factor and the form factor $f_{+}^{K^{+} \pi^{+}}(0)$ contains the $\mathrm{SU}(3)$-breaking quark mass effects. The explicit form of $I\left(m_{K^{+}}, m_{\pi^{+}}\right)$can be found in [11]. With the above estimates for the loop functions and the QCD correction factors, the branching ratio is predicted to be [12]

$$
\operatorname{Br}\left(K^{+} \rightarrow \pi^{+} \nu \bar{\nu}\right)_{\mathrm{SM}}=(9.1 \pm 3.8) \times 10^{-11},
$$

in the SM, where the error is dominated by the uncertainties of the CKM matrix elements.

The effective Lagrangian of the $B^{0}-\overline{B^{0}}$ mixing in the SM is expressed by

$$
\mathcal{L}_{\text {eff }}^{\Delta B=2}=\frac{G_{F}^{2} M_{W}^{2}}{4 \pi^{2}} \bar{d} \gamma^{\mu} P_{L} b \bar{d} \gamma_{\mu} P_{L} b \sum_{i, j=2,3} V_{i 1}^{*} V_{i 3} V_{j 1}^{*} V_{j 3} F_{V}^{W}(i, j) .
$$

Likewise, $\mathcal{L}_{\text {eff }}^{\Delta S=2}$ for the $K^{0}-\overline{K^{0}}$ mixing is obtained by replacing $V_{i 3}$ with $V_{i 2}$, and the $b$-quark operators with the $s$-quark ones, respectively. The loop function $F_{V}^{W}(i, j)$ is given by 9

$$
\begin{aligned}
F_{V}^{W}(i, j)=- & \frac{1}{4} x_{i} x_{j}\left\{\frac{x_{i}^{2}-8 x_{i}+4}{\left(x_{i}-x_{j}\right)\left(x_{i}-1\right)^{2}} \ln x_{i}+\frac{x_{j}^{2}-8 x_{j}+4}{\left(x_{j}-x_{i}\right)\left(x_{j}-1\right)^{2}} \ln x_{j}\right. \\
& \left.-\frac{3}{\left(x_{i}-1\right)\left(x_{j}-1\right)}\right\}, \\
F_{V}^{W}(i, i)=- & \frac{1}{4}\left(\frac{x_{i}}{x_{i}-1}\right)^{2}\left\{x_{i}-11+\frac{4}{x_{i}}+\frac{6 x_{i}}{x_{i}-1} \ln x_{i}\right\},
\end{aligned}
$$

where $x_{i}$ is defined by $x_{i} \equiv m_{u_{i}}^{2} / m_{W}^{2}$. The $B$-meson mixing parameter $x_{d}$ is defined by $x_{d} \equiv \Delta M_{B} / \Gamma_{B}$, where $\Delta M_{B}$ and $\Gamma_{B}$ correspond to the $B$-meson mass difference and the average width of the mass eigenstates, respectively. The mass difference is induced by the above $\Delta B=2$ operator (2.5) and we can express the mixing parameter $x_{d}$ in the SM as

$$
x_{d}=\frac{G_{F}^{2}}{6 \pi^{2}} M_{W}^{2} \frac{M_{B}}{\Gamma_{B}} f_{B}^{2} B_{B}\left|V_{31}^{*} V_{33}\right|^{2} \eta_{B}\left|F_{V}^{W}(3,3)\right|,
$$

where $f_{B}, B_{B}$ and $\eta_{B}$ denote the decay constant of $B^{0}$-meson, the bag parameter of $B^{0}-\overline{B^{0}}$ mixing and the short-distance QCD correction factor, respectively.

For the $K^{0}-\overline{K^{0}}$ system, it is known that the theoretical prediction for the mass difference $\Delta M_{K}$ cannot be given precisely because it receives the large longdistance contributions. On the other hand, the CP-violating parameter $\epsilon_{K}$ is 
dominated by the short-distance contributions which are given by the imaginary part of the same box diagram of the $B^{0}-\overline{B^{0}}$ transition besides the external quark lines. We can express the $\epsilon_{K}$ parameter in the SM as

$$
\begin{aligned}
\epsilon_{K}=-e^{i \pi / 4} & \frac{G_{F}^{2}}{12 \sqrt{2} \pi^{2}} M_{W}^{2} \frac{M_{K}}{\Delta M_{K}} f_{K}^{2} B_{K} \operatorname{Im}\left\{\left(V_{31}^{*} V_{32}\right)^{2} \eta_{K_{33}} F_{V}^{W}(3,3)\right. \\
& \left.+\left(V_{21}^{*} V_{22}\right)^{2} \eta_{K_{22}} F_{V}^{W}(2,2)+2\left(V_{31}^{*} V_{32} V_{21}^{*} V_{22}\right) \eta_{K_{32}} F_{V}^{W}(3,2)\right\}
\end{aligned}
$$

where $f_{K}, B_{K}$ and $\eta_{K_{i j}}$ represent the decay constant, the bag parameter and the QCD correction factors, respectively.

Experimentally, both $x_{d}$ and $\epsilon_{K}$ parameters have been measured as [6]

$$
\begin{aligned}
x_{d} & =0.73 \pm 0.05 \\
\left|\epsilon_{K}\right| & =(2.23 \pm 0.013) \times 10^{-3} .
\end{aligned}
$$

In theoretical estimation of these quantities, non-negligible uncertainties come from the evaluations of the QCD correction factors and the hadronic matrix elements. In our analysis, we adopt the following values:

$$
\eta_{B}=0.55 \pm 0.01[13], \quad \sqrt{B_{B}} f_{B}=(220 \pm 40) \mathrm{MeV} \text { [14], }
$$

for the $x_{d}$ parameter, and

$$
\left.\begin{array}{l}
\eta_{K_{33}}=0.57 \pm 0.01 \\
\eta_{K_{22}}=1.38 \pm 0.20 \\
\eta_{K_{32}}=0.47 \pm 0.04
\end{array}\right\}\left[13, \text { 15], } \quad B_{K}=0.75 \pm 0.15\right. \text { [12]. }
$$

for the $\epsilon_{K}$ parameter.

Next, we consider the new physics contributions to these quantities, $\operatorname{Br}\left(K^{+} \rightarrow\right.$ $\pi^{+} \nu \bar{\nu}$ ) (2.3), $x_{d}$ (2.7), and $\epsilon_{K}$ (2.8). In those class of new physics models which have the same FCNC structure with that of the SM, the effective Lagrangians can be obtained by replacing $D_{W}(i)$ with $D^{\text {new }}(i)$ in (2.1), and $F_{V}^{W}(i, j)$ with $F_{V}^{\text {new }}(i, j)$ in (2.7) and (2.8). Then, the effective Lagrangians of these processes in the new physics sector should have the following forms;

$$
\begin{aligned}
\mathcal{L}_{\text {new }}^{K^{+}} & =\frac{G_{F}}{\sqrt{2}} \frac{2 \alpha\left(m_{Z}\right)}{\pi} \frac{1}{\sin ^{2} \theta_{W}} \bar{\nu} \gamma^{\mu} P_{L} \nu V_{32}^{*} V_{31} \bar{s} \gamma_{\mu} P_{L} d A^{\text {new }}, \\
\mathcal{L}_{\text {new }}^{\Delta B=2} & =\frac{G_{F}^{2} M_{W}^{2}}{4 \pi^{2}} \bar{d} \gamma^{\mu} P_{L} b \bar{d} \gamma_{\mu} P_{L} b\left(V_{31}^{*} V_{33}\right)^{2} B^{\text {new }} \\
\mathcal{L}_{\text {new }}^{\Delta S=2} & =\frac{G_{F}^{2} M_{W}^{2}}{4 \pi^{2}} \bar{d} \gamma^{\mu} P_{L} s \bar{d} \gamma_{\mu} P_{L} s\left(V_{31}^{*} V_{32}\right)^{2} B^{\text {new }} .
\end{aligned}
$$


It should be noticed that the new physics contributions to the $\Delta B=2$ (2.12b) and the $\Delta S=2(2.12 \mathrm{~d})$ processes are expressed by the same quantity $B^{\text {new }}$.

There are two cases in which the effective Lagrangians can be given by the above forms. First, if the contributions from the first two generations do not differ much, i.e.,

$$
\begin{aligned}
D^{\text {new }}(2) & \approx D^{\text {new }}(1), \\
F_{V}^{\text {new }}(i, 1) & \approx F_{V}^{\text {new }}(i, 2),
\end{aligned}
$$

the net contributions from the new physics are written by using the unitarity of the CKM matrix as;

$$
\begin{gathered}
\sum_{i} V_{i 2}^{*} V_{i 1} D^{\text {new }}(i) \approx V_{32}^{*} V_{31}\left\{D^{\text {new }}(3)-D^{\text {new }}(1)\right\} \\
\sum_{i, j} V_{i 1}^{*} V_{i k} V_{j 1}^{*} V_{j k} F_{V}^{\text {new }}(i, j) \approx\left(V_{31}^{*} V_{3 k}\right)^{2}\left\{F_{V}^{\text {new }}(3,3)+F_{V}^{\text {new }}(1,1)\right. \\
\left.-F_{V}^{\text {new }}(3,1)-F_{V}^{\text {new }}(1,3)\right\},
\end{gathered}
$$

for $k=2,3$. We can now define the parameters $A^{\text {new }}$ and $B^{\text {new }}$ as

$$
\begin{aligned}
& A^{\text {new }} \equiv D^{\text {new }}(3)-D^{\text {new }}(1), \\
& B^{\text {new }} \equiv F_{V}^{\text {new }}(3,3)+F_{V}^{\text {new }}(1,1)-F_{V}^{\text {new }}(3,1)-F_{V}^{\text {new }}(1,3) .
\end{aligned}
$$

Second, if the contributions from both the first two generations are negligible as compared with those of the 3rd generation, i.e.,

$$
\begin{aligned}
D^{\text {new }}(3) & \gg D^{\text {new }}(1), D^{\text {new }}(2), \\
F_{V}^{\text {new }}(3,3) & \gg F_{V}^{\text {new }}(1, j), F_{V}^{\text {new }}(2, j), F_{V}^{\text {new }}(3,1), F_{V}^{\text {new }}(3,2),
\end{aligned}
$$

the parameters $A^{\text {new }}$ and $B^{\text {new }}$ become

$$
\begin{aligned}
& A^{\text {new }}=D^{\text {new }}(3), \\
& B^{\text {new }}=F_{V}^{\text {new }}(3,3) .
\end{aligned}
$$

Now, the effects of the new physics contributions to these processes can be evaluated by the following ratios

$$
\begin{aligned}
& R_{1}=\frac{F_{V}^{W}(3,3)+B^{\text {new }}}{F_{V}^{W}(3,3)} \\
& R_{2}=\frac{D_{W}(3)+A^{\text {new }}}{D_{W}(3)} .
\end{aligned}
$$


Once a model of new physics is specified, we can quantitatively estimate the new contributions in terms of $R_{1}$ and $R_{2}$. The parameter $R_{1}$ in (2.18a) has been introduced in [16] to measure the MSSM contributions to the $x_{d}$ and $\epsilon_{K}$ parameters. Both $R_{1}$ and $R_{2}$ parameters converge to unity as the new physics contributions are negligible,

$$
R_{1}, R_{2} \longrightarrow 1 \quad \text { for } \quad A^{\text {new }}, B^{\text {new }} \longrightarrow 0
$$

In the following, we consider the cases where the net contributions from new physics do not exceed those of the SM: $A^{\text {new }}<\left|D_{W}(3)\right|$ and $B^{\text {new }}<\left|F_{V}^{W}(3,3)\right|$. Then we study constraints on $R_{1}, R_{2}$ from experimental results in the region of $0<R_{1}, R_{2}<2$. For instance, in the MSSM and the THDM, predictions are found in the region $0<R_{1}, R_{2}<2$ as shown in Sec. 4 .

\section{Constraints on the new physics contributions to FCNC processes}

If new physics contributions to $x_{d}, \epsilon_{K}$ and $\operatorname{Br}\left(K^{+} \rightarrow \pi^{+} \nu \bar{\nu}\right)$ are sizable, the effects can be detected as deviations of $R_{1}$ and $R_{2}$ from unity. In practice, experimentally measurable quantities are products of the $R_{1}$ or $R_{2}$ by the CKM matrix elements. In the standard parametrization of the CKM matrix, the uncertainty in the CPviolating phase $\delta$ dominates that of the CKM matrix elements [6]. Hence, together with $R_{1}$ and $R_{2}$, we allow $\cos \delta$ to be fitted by the measurements of $x_{d}, \epsilon_{K}$ and $\operatorname{Br}\left(K^{+} \rightarrow \pi^{+} \nu \bar{\nu}\right)$. For this reason, constraints on $R_{1}$ and $R_{2}$ are correlated through $\cos \delta$.

We perform the $\chi^{2}$-fit for two parameters $R_{1}$ and $\cos \delta$ by using experimental data of $x_{d}$ and $\epsilon_{K}$. In our fit, we take into account of the theoretical uncertainties which are given in (2.10), (2.11) and

$$
\left.\begin{array}{rl}
\left|V_{12}\right| & =0.2205 \\
\left|V_{23}\right| & =0.041 \pm 0.003 \\
\left|V_{13} / V_{23}\right| & =0.08 \pm 0.02
\end{array}\right\}[6], \quad m_{t}=175.6 \pm 5.5 \mathrm{GeV} \text { 17, }
$$

where the error of $\left|V_{12}\right|$ can be safely neglected. We find

$$
\left.\begin{array}{l}
\cos \delta=0.36 \pm 0.83 \\
R_{1}=0.93 \pm 0.75
\end{array}\right\} \quad \rho_{\text {corr }}=0.90
$$




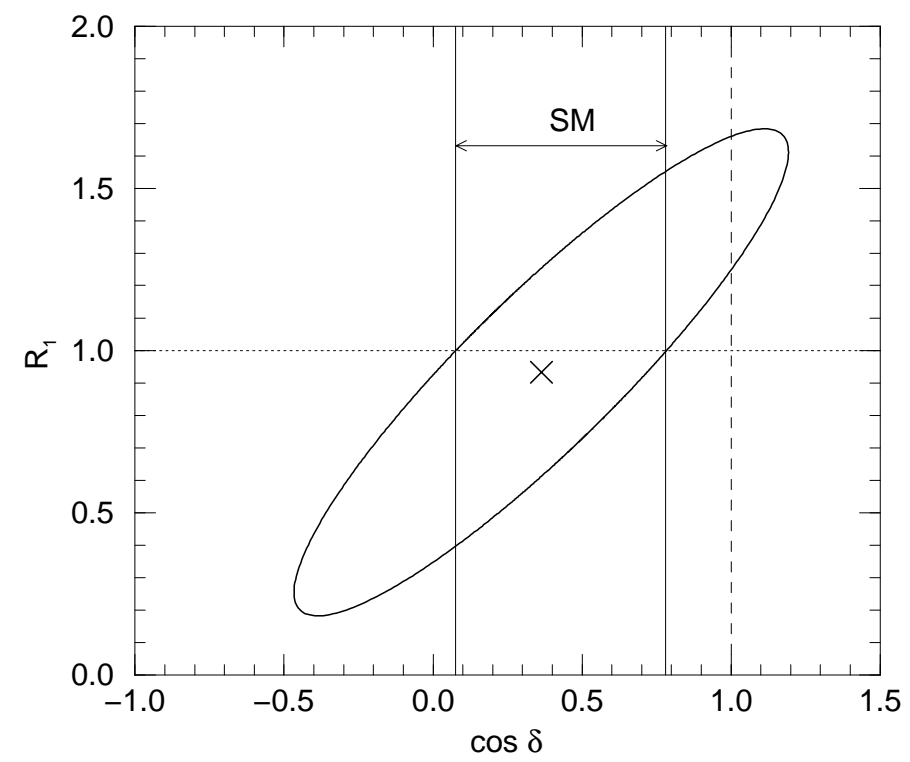

Figure 1: The 1- $\sigma(39 \% \mathrm{CL})$ allowed region from the experimental results of the $B^{0}-\overline{B^{0}}, K^{0}-\overline{K^{0}}$ mixings. The range between the two solid lines is the allowed region of $\cos \delta$ in the $\mathrm{SM}$.

Because of the strong positive correlation between the errors, only the following combination is effectively constrained;

$$
R_{1}=0.61+0.89 \cos \delta \pm 0.33
$$

We show the $1-\sigma(39 \%)$ allowed region of $\cos \delta$ and $R_{1}$ in Fig. 1. In the figure, there is small region which corresponds to $1 \leq \cos \delta$ where the flavor mixing does not obey the CKM mechanism. The range of $\cos \delta$ along the $R_{1}=1$ line is the allowed region of $\cos \delta$ in the SM: $0.08<\cos \delta<0.78$. We can read off from Fig. 1 that the current experimental data of $x_{d}$ and $\epsilon_{K}$ parameters constrain the new physics contributions within $0.18 \lesssim R_{1} \lesssim 1.68$.

Next we examine the constraint on $R_{2}$. Although the recent observation of one candidate event is unsuitable to include in the actual fit, we can expect that the data will be improved in the near future. In the following, we adopt the central value of the SM prediction as the mean value of $\operatorname{Br}\left(K^{+} \rightarrow \pi^{+} \nu \bar{\nu}\right)$ and study consequences of improved measurements. With several more events, the branching fraction can be measured as $\operatorname{Br}\left(K^{+} \rightarrow \pi^{+} \nu \bar{\nu}\right)=(0.9 \pm 0.4) \times 10^{-10}$. 


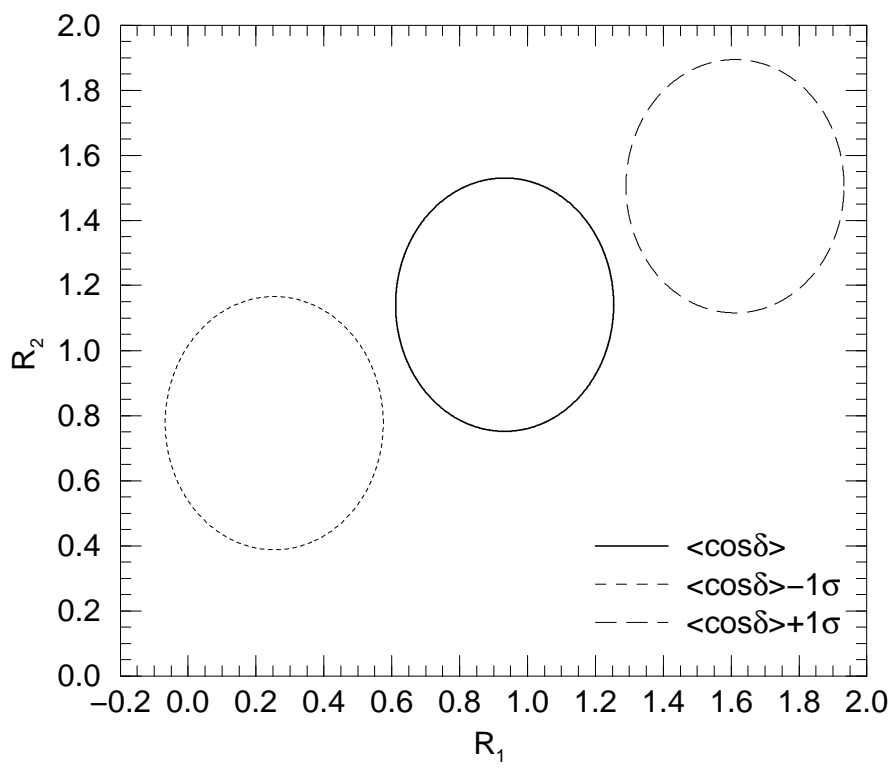

Figure 2: The 1- $\sigma$ allowed regions of $R_{1}, R_{2}$ parameters. Three contours are corresponding to $\cos \delta=0.36$ (solid line), $\cos \delta=-0.47$ (dotted line) and $\cos \delta=1.19$ (dashed line), respectively.

Then the combined result with $x_{d}$ and $\epsilon_{K}$ parameters can be found as

$$
\left.\begin{array}{l}
\cos \delta=0.36 \pm 0.83 \\
R_{1}=0.93 \pm 0.75 \\
R_{2}=1.14 \pm 0.53
\end{array}\right\} \quad \rho_{\text {corr }}=\left(\begin{array}{rrr}
1 & 0.90 & 0.68 \\
& 1 & 0.62 \\
& & 1
\end{array}\right) \text {. }
$$

In Fig. 2, the results are shown on the $R_{1}-R_{2}$ plane for three values of $\cos \delta$; $\cos \delta=0.36$ (mean value), -0.47 (mean value $-1 \sigma$ ) and 1.19 (mean value $+1 \sigma$ ). Using this result, we can discuss about constraints on the new physics contributions to these processes on the $R_{1}-R_{2}$ plane for a given value of $\cos \delta$.

\section{Constraints on MSSM and THDM contribu- tions to the FCNC processes}

Our assumptions on the properties of new physics for FCNC processes in $B$ or $K$ meson systems are naturally satisfied in both the MSSM and the THDM. Predictions on those processes in the contexts of the MSSM and the THDM have been studied in [16, 18, 19, 20] for $B^{0}-\overline{B^{0}}, K^{0}-\overline{K^{0}}$ mixings, and [21, 22, 23] for 
$K^{+} \rightarrow \pi^{+} \nu \bar{\nu}$ process. In this section, we evaluate the $R_{1}, R_{2}$ parameters in both models and find constraints on them from the result in the previous section.

In the MSSM based on $N=1$ supergravity [7], degeneracy of squark masses between the first two generations holds in good approximation. The interaction vertices among down-type quarks $\left(d_{i}\right)$, up-type squarks $\left(\tilde{u}_{j}\right)$ and charginos $(\tilde{\omega})$ are proportional to the CKM matrix elements $V_{i j}$. Since the top-quark mass could induce the large left-right mixing in the $t$-squark sector, one of the $t$-squarks in the mass eigenstates can become lighter than the other squarks. Presence of such a light $t$-squark weakens the unitarity cancellation among the chargino- $u_{j}$-squark exchange diagrams. Therefore the sizable new contributions to the processes may arise from the lighter $t$-squark and chargino exchange diagram.

The MSSM has the physical charged Higgs boson as a consequence of the supersymmetric extension of the Higgs sector. The interactions among the charged Higgs boson and quarks are the same with those of the type II-THDM [8]. The charged Higgs boson interacts with $d_{i}$ and $u_{j}$-quarks through the Yukawa interactions which are proportional to the corresponding quark masses. As a result, the charged Higgs boson contributions to the FCNC processes are dominated by its interaction with the top-quark.

There are other sources of FCNC in the MSSM - the interactions among $d_{i^{-}}$ quark, down-type squarks and neutralinos or the gluino. For $\tan \beta \sim O(1)$, where $\tan \beta$ is the ratio of the vacuum expectation values of two Higgs fields, the left-right mixing in the down-type squark sector is not so large because of the smallness of the down-type quark mass. Furthermore, it has been studied that these diagrams do not give sizable contributions to the FCNC processes for $\tan \beta \lesssim 10$ [19, 23.

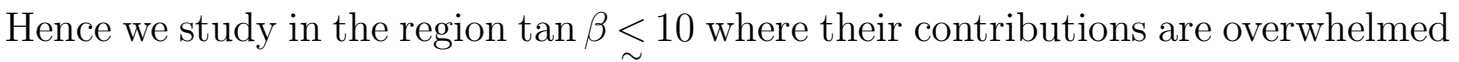
by the $t$-squark-chargino and the charged Higgs boson-top-quark contributions.

The expressions for $R_{1}$ in the MSSM and the THDM can be found in [16]. The MSSM contribution to the decay process $K^{+} \rightarrow \pi^{+} \nu \bar{\nu}$ is expressed by using $D^{\text {new }}$ as follows

$$
D^{\text {new }}(i)=\sum_{m, n, k, \alpha, \beta} D_{C}(i, m, n ; \ell, k ; \alpha, \beta)+D_{H}(i, \ell),
$$

where $D_{C}(i, m, n ; \ell, k ; \alpha, \beta)$ and $D_{H}(i, \ell)$ represent the chargino and the charged Higgs boson contributions, respectively. The chargino contribution $D_{C}$ is given by

$$
D_{C}(i, m, n ; \ell, k ; \alpha, \beta)=D_{C}^{(1)}+D_{C}^{(2)}+D_{C}^{(3)}+D_{C}^{(4)}
$$


and

$$
\begin{aligned}
& D_{C}^{(1)}=-\frac{1}{4}\left(-\frac{1}{2}+\frac{1}{3} \sin ^{2} \theta_{W}\right)\left|F_{i m}^{\alpha}\right|^{2} f_{1}\left(s_{\alpha}, r_{i m}\right), \\
& D_{C}^{(2)}=-\frac{1}{4} F_{i m}^{\alpha *} F_{i m}^{\beta}\left\{B_{\alpha \beta}^{L} f_{2}\left(s_{\alpha}, s_{\beta}, r_{i m}\right)+B_{\alpha \beta}^{R} f_{3}\left(s_{\alpha}, s_{\beta}, r_{i m}\right)\right\}, \\
& D_{C}^{(3)}=-\frac{1}{4} F_{i m}^{\alpha *} F_{i n}^{\alpha} D_{m n}^{i} f_{4}\left(r_{i m}, r_{i n}, s_{\alpha}\right), \\
& D_{C}^{(4)}=\frac{1}{16} F_{i m}^{\alpha} F_{i m}^{\beta *} G_{\ell k}^{\alpha *} G_{\ell k}^{\beta} Y_{1}\left(s_{\alpha}, s_{\beta}, r_{i m}, t_{\ell k}\right),
\end{aligned}
$$

where the indices $(i, \ell)$ denote the squark and slepton generations while $(m, n, k)$ represent two squarks or sleptons for each generation. The indices $(\alpha, \beta)$ stand for the two charginos. The terms $r_{i m}, t_{\ell k}$ and $s_{\alpha}$ are defined by

$$
\begin{aligned}
& r_{11}=r_{21}=\frac{m_{\tilde{u} L}^{2}}{M_{W}^{2}}, \quad r_{12}=r_{22}=\frac{m_{\tilde{u} R}^{2}}{M_{W}^{2}}, \quad r_{3 k}=\frac{m_{\tilde{t} k}^{2}}{M_{W}^{2}}, \\
& t_{11}=t_{21}=t_{31}=\frac{m_{\tilde{e} L}^{2}}{M_{W}^{2}}, \quad t_{12}=t_{22}=t_{32}=\frac{m_{\tilde{e} R}^{2}}{M_{W}^{2}}, \\
& s_{\alpha}=\frac{m_{\tilde{\omega} \alpha}^{2}}{M_{W}^{2}}
\end{aligned}
$$

The coupling constants $F_{i m}^{\alpha}, B_{\alpha \beta}^{L(\text { or } R)}, D_{m n}^{i}$ and $G_{\ell k}^{\alpha}$, and the loop functions $f_{1} \sim f_{4}$ and $Y_{1}$ are explicitly shown in Appendices A and B. By using the unitarity of the CKM matrix and the degeneracy of the squark masses between the first two generations, we obtain

$V_{i 2}^{*} V_{i 1} D_{C}(i, m, n ; \ell, k ; \alpha, \beta)=V_{32}^{*} V_{31}\left\{D_{C}(3, m, n ; \ell, k ; \alpha, \beta)-D_{C}(1, m, n ; \ell, k ; \alpha, \beta)\right\}$,

and the chargino contribution $A^{\text {new }} \equiv A_{C}$ is given by

$$
A_{C} \equiv \sum_{m, n, k, \alpha, \beta}\left\{D_{C}(3, m, n ; \ell, k ; \alpha, \beta)-D_{C}(1, m, n ; \ell, k ; \alpha, \beta)\right\} .
$$

The charged Higgs boson contribution $D_{H}(i, \ell)$ is given by

$$
D_{H}(i, \ell)=D_{H Z}(i)+D_{H H}(i, \ell)+D_{H W}(i, \ell),
$$

and

$$
\begin{aligned}
D_{H Z}(i) & =-\frac{1}{8} x_{i} \cot ^{2} \beta\left[\frac{z_{i}}{\left(z_{i}-1\right)^{2}} \ln z_{i}-\frac{z_{i}}{z_{i}-1}\right], \\
D_{H H}(i, \ell) & =\frac{1}{16} x_{i} \tilde{z}_{\ell} Y_{1}\left(x_{H}, x_{H}, x_{i}, \tilde{z}_{\ell}\right),
\end{aligned}
$$



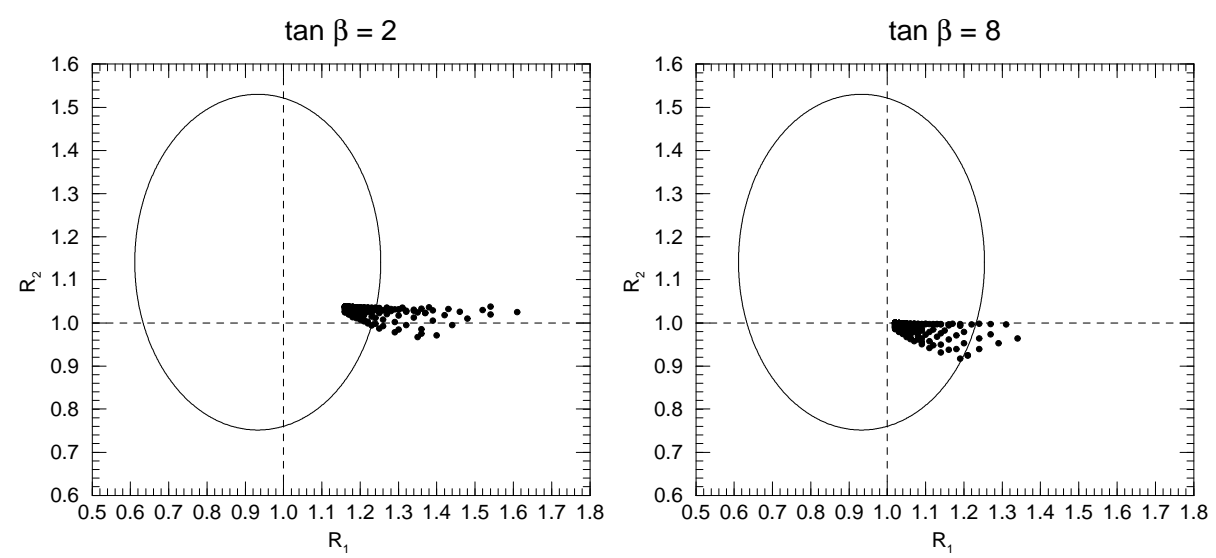

Figure 3: The MSSM contributions to $R_{1}, R_{2}$ parameters for $\tan \beta=2$ (left) and $\tan \beta=8$ (right). The $1-\sigma$ allowed region of $R_{1}, R_{2}$ parameters for $\cos \delta=0.36$ is also shown.

$$
\begin{gathered}
D_{H W}(i, \ell)=\frac{\sqrt{x_{i} \tilde{z}_{\ell}}}{2} Y_{2}\left(x_{H}, 1, x_{i}, \tilde{z}_{\ell}\right)+\frac{1}{8} x_{i} \tilde{z}_{\ell} Y_{1}\left(x_{H}, 1, x_{i}, \tilde{z}_{\ell}\right), \\
x_{H}=\frac{m_{H}^{2}}{m_{W}^{2}}, \quad z_{i}=\frac{m_{u i}^{2}}{m_{H}^{2}}, \quad \tilde{z}_{\ell}=\frac{m_{e \ell}^{2}}{m_{H}^{2}},
\end{gathered}
$$

where the indices $(i, \ell)$ correspond to the quark and lepton generations, respectively. $m_{H}$ being the charged Higgs boson mass and $\beta$ is defined as $\tan \beta \equiv v_{2} / v_{1}$, $v_{1}$ and $v_{2}$ are the vacuum expectation values of the Higgs fields of the hyper-charge $Y=-1 / 2$ and $Y=+1 / 2$, respectively. The loop function $Y_{2}$ is given in Appendix B. Due to the smallness of the Yukawa couplings for light quarks, the top-quark loop functions $(i=3)$ give dominant contributions. Then we can write the charged Higgs contribution as

$$
A_{H} \equiv D_{H}(3, \ell) .
$$

From (4.6) and (4.9), $R_{2}$ in the MSSM is defined as

$$
R_{2} \equiv \frac{D_{W}(3)+A_{C}+A_{H}}{D_{W}(3)} .
$$

The MSSM has several unknown parameters. In order to reduce the number of input parameters in numerical study, we express the soft SUSY breaking scalar masses in the squark and the slepton sectors by a common mass parameter $m_{0}$. Also taking the scalar trilinear coupling $A_{f}$ ( $f$ denotes squarks or sleptons) as $A_{f}=m_{0}$, the MSSM contributions can be evaluated by using four parameters, 


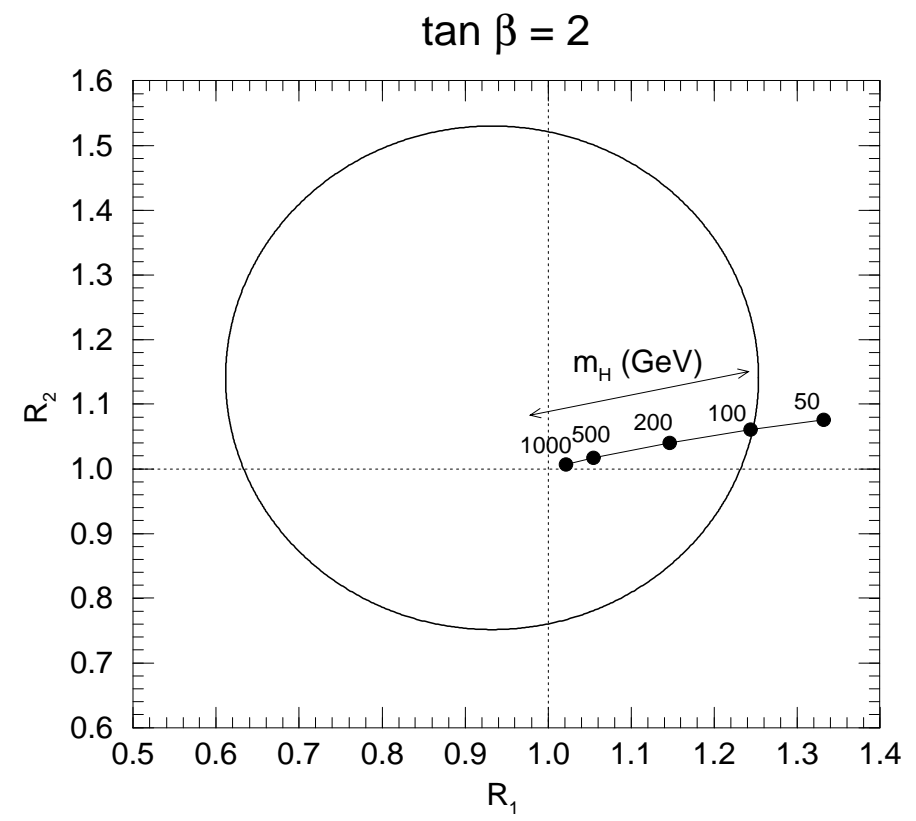

Figure 4: The THDM contributions to $R_{1}, R_{2}$ parameters for $\tan \beta=2$. The $1-\sigma$ allowed region of $R_{1}, R_{2}$ parameters for $\cos \delta=0.36$ is also shown.

$m_{0}, \tan \beta$, the higgsino mass term $\mu$ and the $\mathrm{SU}(2)$ gaugino mass term $m_{2}$. In our study, these parameters are taken to be real. In Fig. 3, we show the MSSM contributions to $R_{1}, R_{2}$ parameters with the constraints on these parameters for $\cos \delta=0.36$. The numerical study was performed in the range of $100 \mathrm{GeV}<$ $m_{0}<1 \mathrm{TeV},|\mu|<200 \mathrm{GeV}$ and $m_{2}=200 \mathrm{GeV}$ for $\tan \beta=2$ and 8 . We fixed the charged Higgs boson mass at $m_{H}=200 \mathrm{GeV}$. This is the reason why the MSSM contributions do not converge to $R_{1}=1$ in Fig. 3. We take into account the recent estimation of lower mass limits for lighter $t$-squark and lighter chargino [24]: $80 \mathrm{GeV} \leq m_{\tilde{t} 1}$ and $91 \mathrm{GeV} \leq m_{\tilde{\omega} 1}$. The MSSM contribution to $R_{1}$ interferes with that of the SM constructively [16, 19, 25]. On the other hand, the contribution to $R_{2}$ interferes with that of the SM both constructively and destructively.

The THDM contribution to $R_{2}$ is given by setting $D_{C}=0$ in (4.10):

$$
R_{2} \equiv \frac{D_{W}(3)+A_{H}}{D_{W}(3)} .
$$

We show in Fig. 1 the charged Higgs contribution to $R_{1}, R_{2}$ parameters for $\tan \beta=$ 2 and $\cos \delta=0.36$. Contrary to the case of the MSSM, the THDM contribution constructively interferes with the SM contribution for both $R_{1}$ and $R_{2}$. Here we 
show the case of $\tan \beta=2$ only. The Yukawa interaction between the top-quark and the charged Higgs boson is proportional to $1 / \tan ^{2} \beta$. Thus constraints on the THDM contribution to these quantities are weakened together with the increase of $\tan \beta$.

\section{Summary}

We have studied impacts on searching for signatures of new physics beyond the $\mathrm{SM}$ from some FCNC processes $-B^{0}-\overline{B^{0}}, K^{0}-\overline{K^{0}}$ mixings and the rare decay $K^{+} \rightarrow \pi^{+} \nu \bar{\nu}$. For a certain class of models of new physics, we showed the extra contributions to the FCNC processes can be parametrized by its ratio to the SM contribution with the common CKM matrix elements. Two parameters $R_{1}$ and $R_{2}$ were introduced to estimate the new physics contributions to $B^{0}-\overline{B^{0}}, K^{0}-\overline{K^{0}}$ mixings and $K^{+} \rightarrow \pi^{+} \nu \bar{\nu}$ decay, respectively. Then the new physics contributions are evaluated from experimental data by using these parameters and $\cos \delta$.

Taking account of both experimental and theoretical uncertainties for the $B^{0}-\overline{B^{0}}$ and $K^{0}-\overline{K^{0}}$ mixings, constraints on the new physics contribution to $R_{1}$ and $\cos \delta$ were shown: the allowed range of $R_{1}$ is $0.18 \underset{\sim}{\sim} R_{1} \underset{\sim}{ } 1.68$. With the assumption that the future data of $\operatorname{Br}\left(K^{+} \rightarrow \pi^{+} \nu \bar{\nu}\right)$ will be close to the SM prediction, constraints on $\cos \delta, R_{1}$ and $R_{2}$ were found. The results were applied to the MSSM and the THDM contributions to those processes. Our study will become useful if the measured value of $\operatorname{Br}\left(K^{+} \rightarrow \pi^{+} \nu \bar{\nu}\right)$ is close to the $\mathrm{SM}$ prediction. Then, we may expect to obtain the constraints on the new physics parameters through $R_{1}$ and $R_{2}$.

\section{Acknowledgment}

The author thanks to K. Hagiwara, Y. Okada and Y. Shimizu for discussions and comments. This work is supported in part by Grant-in-Aid for Scientific Research from the Ministry of Education, Science and Culture of Japan.

Note added: While we were preparing this paper, we found ref. [23], where a parametrization of new physics contributions to $K^{+} \rightarrow \pi^{+} \nu \bar{\nu}$ is proposed and consequences of the SUSY-SM are studied. Their parametrization is similar to ours besides that they define the parameter $R_{2}$ (denoted as $r_{K}$ in their paper) 
as a complex parameter. Our result of the MSSM contributions to the decay process is consistent with theirs. Correlation between the MSSM contributions to $K^{+} \rightarrow \pi^{+} \nu \bar{\nu}$ and the $x_{d}, \epsilon_{K}$ parameters are not discussed in their paper.

\section{Appendix A: Masses and coupling constants in the MSSM}

In this appendix, we give the explicit forms of coupling constants in (4.3). We first introduce the squark, slepton and chargino masses. The squark masses in the first and the second generations are given by

$$
\begin{aligned}
& m_{\tilde{u} L}^{2}=m_{\tilde{c} L}^{2}=m_{Q}^{2}+\cos 2 \beta\left(\frac{1}{2}-\frac{2}{3} \sin ^{2} \theta_{W}\right) m_{Z}^{2}, \\
& m_{\tilde{u} R}^{2}=m_{\tilde{c} R}^{2}=m_{U}^{2}+\frac{2}{3} \cos 2 \beta \sin ^{2} \theta_{W} m_{Z}^{2}
\end{aligned}
$$

where the corresponding quark masses can be safely neglected. The parameters $m_{Q}$ and $m_{U}$ are the soft SUSY breaking squark masses for the SU(2) doublet and the singlet, respectively. The angle $\beta$ is defined by $\tan \beta=v_{2} / v_{1}$, where $v_{1}, v_{2}$ are the vacuum expectation values of the Higgs doublets. The squared mass matrix for the $t$-squark is given by

$$
M_{\tilde{t}}^{2}=\left(\begin{array}{cc}
m_{\tilde{u} L}^{2}+m_{t}^{2} & -m_{t}\left(\mu \cot \beta+A_{t}\right) \\
-m_{t}\left(\mu \cot \beta+A_{t}\right) & m_{\tilde{u} R}^{2}+m_{t}^{2}
\end{array}\right),
$$

where the dimensionful parameter $A_{t}$ and $\mu$ denote the scalar trilinear coupling and the higgsino mass term, respectively. The mass matrix $M_{\tilde{t}}^{2}$ can be diagonalized by using the unitary matrix $S_{t}$,

$$
S_{t} M_{\tilde{t}}^{2} S_{t}^{\dagger}=\operatorname{diag}\left(m_{\tilde{t} 1}^{2}, m_{\tilde{t} 2}^{2}\right) \quad\left(m_{\tilde{t} 1}^{2}<m_{\tilde{t} 2}^{2}\right)
$$

The charged slepton masses are given by

$$
\begin{aligned}
& m_{\tilde{e} L}^{2}=m_{\tilde{\mu} L}^{2}=m_{\tilde{\tau} L}^{2}=m_{L}^{2}+\cos 2 \beta\left(-\frac{1}{2}+\sin ^{2} \theta_{W}\right) m_{Z}^{2}, \\
& m_{\tilde{e} R}^{2}=m_{\tilde{\mu} R}^{2}=m_{\tilde{\tau} R}^{2}=m_{E}^{2}-\cos 2 \beta \sin ^{2} \theta_{W} m_{Z}^{2},
\end{aligned}
$$

where $m_{L}$ and $m_{E}$ represent the soft SUSY breaking slepton masses for the SU(2) doublet and the singlet, respectively. We neglected the corresponding charged lepton masses. 
The chargino mass matrix is given by

$$
M^{-}=\left(\begin{array}{cc}
m_{2} & \sqrt{2} M_{W} \cos \beta \\
\sqrt{2} M_{W} \sin \beta & \mu
\end{array}\right),
$$

where $m_{2}$ is the $\mathrm{SU}(2)$ gaugino mass. We can obtain the mass eigenstates by using two unitary matrices $C_{R}$ and $C_{L}$;

$$
C_{R}^{\dagger} M^{-} C_{L}=\operatorname{diag}\left(\tilde{m}_{\omega 1}, \tilde{m}_{\omega 2}\right) \quad\left(\tilde{m}_{\omega 1}<\tilde{m}_{\omega 2}\right) .
$$

The couplings constants $F_{i j}^{\alpha}, B_{\alpha \beta}^{k}(k=L, R), D_{\ell m}^{i}$ and $G_{i j}^{\alpha}$ in (4.3) are given as;

$$
\left.\begin{array}{c}
F_{11}^{\alpha}=F_{21}^{\alpha}=\sqrt{2} C_{R 1 \alpha}^{*}, \quad F_{12}^{\alpha}=F_{22}^{\alpha}=0, \\
F_{3 j}^{\alpha}=\sqrt{2} C_{R 1 \alpha}^{*} S_{t j 1}-\frac{m_{t}}{M_{W} \sin \beta} C_{R 2 \alpha}^{*} S_{t j 2}
\end{array}\right\},
$$

where the expressions for the first two generation of squarks in (A.9) can be obtained by replacing $S_{t}$ with the unit matrix.

\section{Appendix B: Loop functions}

The loop function $f_{1} \sim f_{4}$ in (4.3a) $\sim 4.3 \mathrm{~d}$ ) are given as;

$$
\begin{array}{r}
f_{1}(x, y)=\frac{1}{4}+\frac{1}{2} \frac{x}{x-y}-\frac{1}{2}\left\{\ln y+\left(\frac{x}{x-y}\right)^{2}(\ln x-\ln y)\right\}, \\
f_{2}\left(x_{i}, x_{j}, y\right)=-\sqrt{x_{i} x_{j}}\left\{\frac{x_{i} \ln x_{i}}{\left(x_{i}-x_{j}\right)\left(x_{i}-y\right)}+\frac{x_{j} \ln x_{j}}{\left(x_{j}-x_{i}\right)\left(x_{j}-y\right)}\right. \\
\left.+\frac{y \ln y}{\left(y-x_{i}\right)\left(y-x_{j}\right)}\right\},
\end{array}
$$




$$
\begin{aligned}
& f_{2}(x, x, y)=-\frac{x}{y-x}\left\{\frac{y}{y-x}(\ln y-\ln x)-1\right\} \\
& f_{3}\left(x_{i}, x_{j}, y\right)=\frac{1}{2}\left\{\frac{x_{i}^{2} \ln x_{i}}{\left(x_{i}-x_{j}\right)\left(x_{i}-y\right)}\right.+\frac{x_{j}^{2} \ln x_{j}}{\left(x_{j}-x_{i}\right)\left(x_{j}-y\right)} \\
&\left.+\frac{y^{2} \ln y}{\left(y-x_{i}\right)\left(y-x_{j}\right)}\right\}-\frac{1}{4}, \\
& f_{3}(x, x, y)=\frac{1}{2}\left\{\left(\frac{y}{y-x}\right)^{2}(\ln y-\ln x)+\ln x-\frac{x}{y-x}\right\}-\frac{1}{4}, \\
& f_{4}\left(x_{i}, x_{j}, y\right)=f_{3}\left(x_{i}, x_{j}, y\right)-\frac{1}{2}, \\
& f_{4}(x, x, y)=f_{3}(x, x, y)-\frac{1}{2} .
\end{aligned}
$$

The loop functions $Y_{1}, Y_{2}$ which come from the box type diagrams are given by;

$$
\begin{gathered}
Y_{1}\left(r_{\alpha}, r_{\beta}, s_{i}, s_{j}\right)=\frac{r_{\alpha}^{2}}{\left(r_{\beta}-r_{\alpha}\right)\left(s_{i}-r_{\alpha}\right)\left(s_{j}-r_{\alpha}\right)} \ln r_{\alpha}+\frac{r_{\beta}^{2}}{\left(r_{\alpha}-r_{\beta}\right)\left(s_{i}-r_{\beta}\right)\left(s_{j}-r_{\beta}\right)} \ln r_{\beta} \\
+\frac{s_{i}^{2}}{\left(r_{\alpha}-s_{i}\right)\left(r_{\beta}-s_{i}\right)\left(s_{j}-s_{i}\right)} \ln s_{i}+\frac{s_{j}^{2}}{\left(r_{\alpha}-s_{j}\right)\left(r_{\beta}-s_{j}\right)\left(s_{i}-s_{j}\right)} \ln s_{j}
\end{gathered}
$$$$
Y_{1}\left(r_{\alpha}, r_{\alpha}, s_{i}, s_{j}\right)=\frac{r_{\alpha}\left(s_{i}+s_{j}\right)-2 s_{i} s_{j}}{\left(s_{i}-r_{\alpha}\right)^{2}\left(s_{j}-r_{\alpha}\right)^{2}} r_{\alpha} \ln r_{\alpha}-\frac{r_{\alpha}}{\left(s_{i}-r_{\alpha}\right)\left(s_{j}-r_{\alpha}\right)}
$$$$
+\frac{s_{i}^{2}}{\left(r_{\alpha}-s_{i}\right)^{2}\left(s_{j}-s_{i}\right)} \ln s_{i}+\frac{s_{j}^{2}}{\left(r_{\alpha}-s_{j}\right)^{2}\left(s_{i}-s_{j}\right)} \ln s_{j},
$$

$$
\begin{aligned}
Y_{1}\left(r_{\alpha}, r_{\beta}, s_{i}, s_{i}\right)= & \frac{r_{\alpha}^{2}}{\left(r_{\beta}-r_{\alpha}\right)\left(s_{i}-r_{\alpha}\right)^{2}} \ln r_{\alpha}+\frac{r_{\beta}^{2}}{\left(r_{\alpha}-r_{\beta}\right)\left(s_{i}-r_{\beta}\right)^{2}} \ln r_{\beta} \\
& +\frac{\left(r_{\alpha}+r_{\beta}\right) s_{i}-2 r_{\alpha} r_{\beta}}{\left(r_{\alpha}-s_{i}\right)^{2}\left(r_{\beta}-s_{i}\right)^{2}} s_{i} \ln s_{i}-\frac{s_{i}}{\left(r_{\alpha}-s_{i}\right)\left(r_{\beta}-s_{i}\right)}
\end{aligned}
$$

$Y_{1}\left(r_{\alpha}, r_{\alpha}, s_{i}, s_{i}\right)=-\frac{2 r_{\alpha} s_{i}}{\left(s_{i}-r_{\alpha}\right)^{3}} \ln r_{\alpha}-\frac{2 r_{\alpha} s_{i}}{\left(r_{\alpha}-s_{i}\right)^{3}} \ln s_{i}-\frac{r_{\alpha}+s_{i}}{\left(r_{\alpha}-s_{i}\right)^{2}}$

$$
Y_{2}\left(r_{\alpha}, r_{\beta}, s_{i}, s_{j}\right)=\sqrt{s_{i} s_{j}}\left[\frac{r_{\alpha}}{\left(r_{\beta}-r_{\alpha}\right)\left(s_{i}-r_{\alpha}\right)\left(s_{j}-r_{\alpha}\right)} \ln r_{\alpha}+\frac{r_{\beta}}{\left(r_{\alpha}-r_{\beta}\right)\left(s_{i}-r_{\beta}\right)\left(s_{j}-r_{\beta}\right)} \ln r_{\beta}\right.
$$




$$
\begin{aligned}
& \left.+\frac{s_{i}}{\left(r_{\alpha}-s_{i}\right)\left(r_{\beta}-s_{i}\right)\left(s_{j}-s_{i}\right)} \ln s_{i}+\frac{s_{j}}{\left(r_{\alpha}-s_{j}\right)\left(r_{\beta}-s_{j}\right)\left(s_{i}-s_{j}\right)} \ln s_{j}\right], \\
& Y_{2}\left(r_{\alpha}, r_{\alpha}, s_{i}, s_{j}\right)=\sqrt{s_{i} s_{j}}\left[\frac{r_{\alpha}^{2}-s_{i} s_{j}}{\left(s_{i}-r_{\alpha}\right)^{2}\left(s_{j}-r_{\alpha}\right)^{2}} \ln r_{\alpha}-\frac{1}{\left(s_{i}-r_{\alpha}\right)\left(s_{j}-r_{\alpha}\right)}\right. \\
& \left.+\frac{s_{i}}{\left(r_{\alpha}-s_{i}\right)^{2}\left(s_{j}-s_{i}\right)} \ln s_{i}+\frac{s_{j}}{\left(r_{\alpha}-s_{j}\right)^{2}\left(s_{i}-s_{j}\right)} \ln s_{j}\right], \\
& Y_{2}\left(r_{\alpha}, r_{\beta}, s_{i}, s_{i}\right)=s_{i}\left[\frac{r_{\alpha}}{\left(r_{\beta}-r_{\alpha}\right)\left(s_{i}-r_{\alpha}\right)^{2}} \ln r_{\alpha}+\frac{r_{\beta}}{\left(r_{\alpha}-r_{\beta}\right)\left(s_{i}-r_{\beta}\right)^{2}} \ln r_{\beta}\right. \\
& \left.+\frac{s_{i}^{2}-r_{\alpha} r_{\beta}}{\left(r_{\alpha}-s_{i}\right)^{2}\left(r_{\beta}-s_{i}\right)^{2}} \ln s_{i}-\frac{1}{\left(r_{\alpha}-s_{i}\right)\left(r_{\beta}-s_{i}\right)}\right] \text {, } \\
& Y_{2}\left(r_{\alpha}, r_{\alpha}, s_{i}, s_{i}\right)=s_{i}\left[-\frac{r_{\alpha}+s_{i}}{\left(s_{i}-r_{\alpha}\right)^{3}} \ln r_{\alpha}-\frac{r_{\alpha}+s_{i}}{\left(r_{\alpha}-s_{i}\right)^{3}} \ln s_{i}-\frac{2}{\left(r_{\alpha}-s_{i}\right)^{2}}\right] .
\end{aligned}
$$




\section{References}

[1] G. Buchalla, A.J. Buras and M.E. Lautenbacher, Rev. Mod. Phys. 68 (1996) 1125 .

[2] J. Ellis and J.S. Hagelin, Nucl. Phys. B217 (1983) 189;

D. Rein and L.M. Sehgal, Phys. Rev. D39 (1989) 3325;

J.S. Hagelin and L.S. Littenberg, Prog. Part. Nucl. Phys. 23 (1989) 1;

C.Q. Gang, I.J. Hsu and Y.C. Lin, Phys. Lett. B355 (1995) 569;

S. Fajfer, Nuov. Cim. A110 (1997) 397.

[3] A.J. Buras, Phys. Lett. B333 (1994) 476;

G. Buchalla and A.J. Buras, Nucl. Phys. B412 (1994) 106;

G. Buchalla and A.J. Buras, Phys. Lett. B333 (1994) 221.

[4] G. Buchalla and A.J. Buras, Phys. Rev. D54 (1996) 6782.

[5] E787 collaboration, Phys. Rev. Lett. 79 (1997) 2204.

[6] Particle Data Group, R.M. Barnett et al., Phys. Rev. D54 (1996) 1.

[7] For reviews, see, H.P. Nilles, Phys. Rep. 110 (1984) 1, H.E. Haber and G.L. Kane, Phys. Rep. 117 (1985) 75.

[8] See, e.g., J.F. Gunion, H.E. Haber, G.L. Kane and S. Dawson, The Higgs Hunter's Guide, Addison-Wesley, (1990) and references therein.

[9] T. Inami and C.S. Lim, Prog. Theor. Phys. 65 (1981) 297; (E) 1772.

[10] G. Buchalla and A.J. Buras, Nucl. Phys. B400 (1993) 225.

[11] W.J. Marciano and Z. Parsa, Phys. Rev. D53 (1996) R1.

[12] A.J. Buras and R. Fleischer, hep-ph/9704376;

A.J. Buras, hep-ph/9711217.

[13] A.J. Buras, M. Jamin and P.H. Weisz, Nucl. Phys. B347 (1990) 491.

[14] A. Abada, et al., Nucl. Phys. B376 (1992) 172. A. Abada, LPTHE Orsay$94 / 57$. 
[15] S. Herrlich and U. Nierste, Nucl. Phys. B419 (1994) 292;

S. Herrlich and U. Nierste, Phys. Rev. D52 (1995) 6505, Nucl. Phys. B476 (1996) 27.

[16] G.C. Branco, G.C. Cho, Y. Kizukuri and N. Oshimo, Phys. Lett. B337 (1994) 316; Nucl. Phys. B449 (1995) 483.

[17] CDF Collaboration, J. Lys, talk at ICHEP96, in Proc. of ICHEP96, (ed) Z. Ajduk and A.K. Wroblewski, World Scentific, (1997);

D0 Collaboration, S. Protopopescu, talk at ICHEP96, in the proceedings.

P. Tipton, talk at ICHEP96, in the proceedings.

[18] T. Kurimoto, Phys. Rev. D39 (1989) 3447;

S. Bertolini, F. Borzumati, A. Masiero and G. Ridolfi, Nucl. Phys. B353 (1991) 591;

G. Couture and H. König, Z. Phys. C69 (1996) 499.

[19] T. Goto, T. Nihei and Y. Okada, Phys. Rev. D53 (1996) 5233; (E) D54 (1996) 5904.

[20] L.F. Abott, P. Sikivie and M.B. Wise, Phys. Rev. D21 (1980) 1393.

[21] S. Bertolini and A. Masiero, Phys. Lett. B174 (1986) 343;

B. Mukhopadhyaya and A. Raychaudhuri, Phys. Lett. B189 (1987) 203;

I.I. Bigi and F. Gabbiani, Nucl. Phys. B367 (1991) 3;

G. Couture and H. König, Z. Phys. C69 (1995) 167;

Y. Nir and M.P. Worah, hep-ph/9711215.

[22] A.J. Buras, P. Krawczyk, M.E. Lautenbacher and C. Salazar, Nucl. Phys. B337 (1990) 284.

[23] A.J. Buras, A. Romanio and L. Silvestrini, hep-ph/9712398.

[24] P. Janot, talk given at International Europhysics Conference on High Energy Physics, Jerusalem 1997.

[25] T. Kurimoto, Mod. Phys. Lett. A10 (1995) 1577. 